\title{
Los renacuajos de los anuros (Amphibia) altoandinos de Venezuela: Morfología externa y claves
}

\author{
Abraham Mijares-Urrutia' \\ Centro de Investigaciones en Ecología y Zonas Áridas (CIEZA). Universidad Francisco de Miranda, Apartado 7506, Coro \\ 410I-A, Venezuela. Correo-electrónico: amijares@funflc.org.ve
}

\begin{abstract}
A literature review and examination of several lots of tadpoles representative of the high Venezuelan Andes species (at least one species per genus) allowed identification of 41 useful characters. These are presented (with some illustrations) in a detailed description of their external morphology. An gle measurements (related to body longitudinal axis) are recommended to describe tail tip shape and lo cation of oral apparatuses. Additionally, ninet een meristic data were used to assert morphological proportions. The identification key is based exclusively on external morphology (presence/absence, size, shape, number, location, etc.), that remains mostly unmodified in larval early and int ermediate development. The key allows identification to genus for frogs and toads that occur in the region (six families, 11 genera). As a model of this proposal, the tadpole of Hyla pelidna is described and illustrated for the fir st time; it fits the larval characteristics of the $H$. labialis group (keratodont rows formula $1 / 2$ or $2 / 2$, marginal papillae in multiple rows, rostral gap present, dextral cloacal opening abdominal [separated from the ventral fin], caudal fins widest than caudal musculature at tail midlength, narial opening with fleshy, smooth, not protuberant rim). This is a first effort to establish an anuran larval terminology in Spanish.
\end{abstract}

Key words: Larval morphology, Hyla pelidna, Anura, taxonomy, Andes, Venezuela, key.

La distribución de la herpetofauna altoandina ha sido caracterizada principalmente con base enaquellas especies que habitanen alturas iguales o superiores a los mil metros (Duellman 1979, Lynch 1986), siendo estas, en gran parte, endémicas a los ecosistemas típicos que habitan a partir de dichas elevaciones. En este sentido, corresponde a los anfibios anuros la mayor contribución en diversidad de especies (Péfaur \& Pascual 1982). Los anuros de los altos Andes de Venezuela representan una parte muy importante de la anfibiofauna nativa del país, estimándose en 60 el número de especies actualmente registradas, que se encuentran en esta región altoandina; lo que significa algo menos de un tercio de las 240 especies de anuros venezolanos conocidos hasta el presente (La Marca 1995).
Los renacuajos ofrecen una excelente y valiosísima fuente de caracteres para la identificación de las especies de anuros (Van Dijk 1966). Un hecho destacado es que pueden ser tratados como vertebrados de "vida libre", puesto que estos se enfrentan a las mismas presiones medioambientales que cualquier animal acuático; con la excepción de no reproducirse, estos se mueven, crecen y alimentan (Wassersug 1973), jugando un papel protagónico en la cadena trófica y flujo de energía del medio donde se encuentren, mostrando frecuentemente adaptaciones morfológicas (a veces extremas y específicas) al mismo, lo que los convierte en sujetos de gran interés en la investigación en ecología y evolución, y además, a pesar de la controversia alrededor del uso de los caracteres larvarios en la sistemática de anuros (Donnelly $e t$ 
al. 1990, y otras referencias citadas por ellos), es innegable que los renacuajos si tienen caracteres filogenéticamente informativos (Duellman \& Trueb 1982-1986, Donnelly et al. 1990, Inger 1992). Sin embargo, a pesar de lo expresado arriba, los renacuajos han sido escasamente tomados en cuenta en los diversos estudios sobre anuros que se han llevado a cabo en la región altoandina venezolana (ver revisión general de la bibliografía en La Marca 1992), y una prueba de esto es que, de las 47 especies con etapa de larvas de vida libre registradas en esta región, sólo han sido publicadas las descripciones de las larvas de apenas 16 especies: cinco del género Hyla (Donoso-Barros \& León 1972, Duellman 1972, Rada 1981, La Marca 1985b, Mijares-Urrutia 1990a, 1992), ocho del género Nephelobates (La Marca 1985a,LaMarca\& Mijares 1988, MijaresUrrutia \& La Marca 1997), tres del género Mannophryne (La Marca 1984, 1989, 1994) y uno, respectivamente, de Aromobates (Myers et al. 1991), Bufo (Kenny 1969), Centrolene (Mijares-Urrutia 1990b), Colostethus (MijaresUrrutia 1991), Flectonotus (Duellman \& Gray 1983), Hyalinobatrachium (Starrett 1960), Leptodactylus (Duellman 1978); y Scinax (Hero \& Mijares-Urrutia 1995). Dos géneros, Eleutherodactylus(Leptodactylidae), 13 especies andinas) y Gastrotheca (Hylidae, dos especies andinas) presentan desarrollo directo, es decir, el embrión se transforma directamente en unjuvenil, sin cumplircon laetapade renacuajo propiamente, por lo que, naturalmente, no son de interés para este estudio.

En este trabajo se presentan los resultados de una revisión de las referencias pertinentes al tema y del examen de una serie de lotes de larvas representativas (al menos una especie de cada género) de la anurofauna andina venezolana, de la que se extrajo una extensa lista de los rasgos morfológicos externos más notables para la caracterización e identificación de las larvas de las especies de anuros de esta amplia zona del occidente venezolano, incluyendo también las respectivas claves para la ubicación de los renacuajos al menos hasta los niveles de familia, género $y$, cuando se aplica, a nivel de grupos de especies. También se incluyen algunas ilustraciones explicativas donde se esbozan esquemáticamente la mayoría de los diversos caracteres y medidas necesarias para la reseña de las larvas de anuros. Adicionalmente, y a manera de modelo, se incluye la descripción original del renacuajo de Hyla pelidna (Hylidae).

Finalmente, y como consecuencia natural, se quiere destacarqueel presente trabajoes la primera iniciativa para una propuesta de terminología en idioma español de la morfología externa larvaria partiendo de las observaciones de los renacuajos de los anuros de los Andes venezolanos.

\section{MATERIALES Y MÉTODOS}

Para este trabajo se han examinado los renacuajos de casi todas las especies existentes hasta el presente en la región altoandina ( $\mathrm{La}$ Marca 1984, 1985a-b, 1989; 1994, La Marca \& Mijares 1988, Mijares-Urrutia 1990a-b, 1991, 1992, Mijares-Urrutia \& La Marca 1997). En la clave se emplean exclusivamente caracteres morfológicos (presencia/ausencia, forma, tamaño, cantidad, posición, etc.), no modificables o, cuando menos ligeramente, a lo largo de los estadios pre y prometamórficos del renacuajo (con las excepciones señaladas más adelante). La definición de estos tipos de estadios de desarrollo la da Altig (1970: 186), de la manera siguiente: premetamórfico "estadios de desarrollo entre el momento que el opérculo se cierra y las yemas de las patas posteriores aparecen", prometamórfico "estadios de desarrollo comprendidos entre el momentoen que las yemas delas patas posteriores aparecen y las patas anteriores afloran"'(traducción libre del inglés por el autor). Es necesario aclarar que las definiciones antes dadas son empleadas con un significado inverso a la propuesta de Lavilla \& Rouges (1992) y a conveniencia en el sentidoestrictamente de la morfología comparada y no en términos de desarrollo o metamórficos según los define Echeverría (1990).

En la terminología y caracteres morfológicos externos empleados se sigue una combinación de 
las propuestas dadas por Altig (1970), Johnston \& Altig (1986), Altig \& Johnston (1989), Lavil la (1988) y Van Dijk (1966), además de algunas propuestas nuevas. Para caracteres más específicos, se utilizó a Channing (1984) y Channing et al. (1988) para la configuración del borde de la abertura narinal. Para la anotación de la fórmula de filas de queratodontes se sigue preferentemente a Altig (1970) y Altig \& Johnston (1986), adoptando con cautela las modificaciones propuestas por Dubois (1994), sin embargo, con esteúltimoautor existen diferencias de apreciación sobre ciertas consideraciones morfológicas que no compartimos (por ejemplo, el considerar los queratodontes situados en los ángulos y periferia del disco oral como parte de las filas de queratodontes), pero que en principio no afectan en nada el propósito de nuestro trabajo.

Las medidas se tomaron con un vernier $( \pm 0.1$ mm de apreciación) y un micrómetro ocular en una lupa de disección. Para las medidas se tomó como modelo las hechas por Lavilla \& Scrocchi (1986), modificadas de lamanera siguiente: largo total (ltt), largo del cuerpo (lcp), ancho del cuerpo a nivel del espiráculo (ccp), ancho del cuerpo a nivel de las narinas (ccn), ancho porción final del cuerpo (ccf, esta medida se hará al nivel donde llegue la misma distancia narina-punta del hocico, pero esta vez partiendo desde el final del cuerpo), altura del cuerpo a nivel del espiráculo (acp), largo de la cola (lcl), altura total de la cola a la mitad de su longitud (acl), altura aleta caudal dorsal a la mitad de la longitud caudal (aad), altura aleta caudal ventral a la mitad de la longitud caudal (aav), altura musculatura caudal a la mitad de la longitud caudal (amc), distancia espiráculopuntadel hocico (deh), distancia espiráculo-dorso (ded), anchodiscooral(cdo), distanciainterorbital (dio), distancia ojo-punta del hocico (doh), diámetro horizontal del ojo (fh), distancia ojonarina(don), distancia internarinal (din)(verFigs. 1, 2 y 3). No fueron tomadas en cuenta: ancho del cuerpo a nivel de las narinas, ancho del cuerpo a nivel de los ojos, distancia extranarinal, distancia extraocular, ancho del claro rostral y distancia espiráculo-posterior. La mayoría de los rasgos orales se ilustran en la Fig. 6. Las larvas fueron identificadas por haberse desarrollado parcial o totalmente en ejemplares identificables por características adultas, haber sido recolectados junto o asociados a adultos, la distribución geográfica característica de la especie, o una combinación de todos o algunos de estos aspectos. Los estadios de desarroll o larvarios se establecieron de acuerdo a la Cuadro de Gosner (1960).

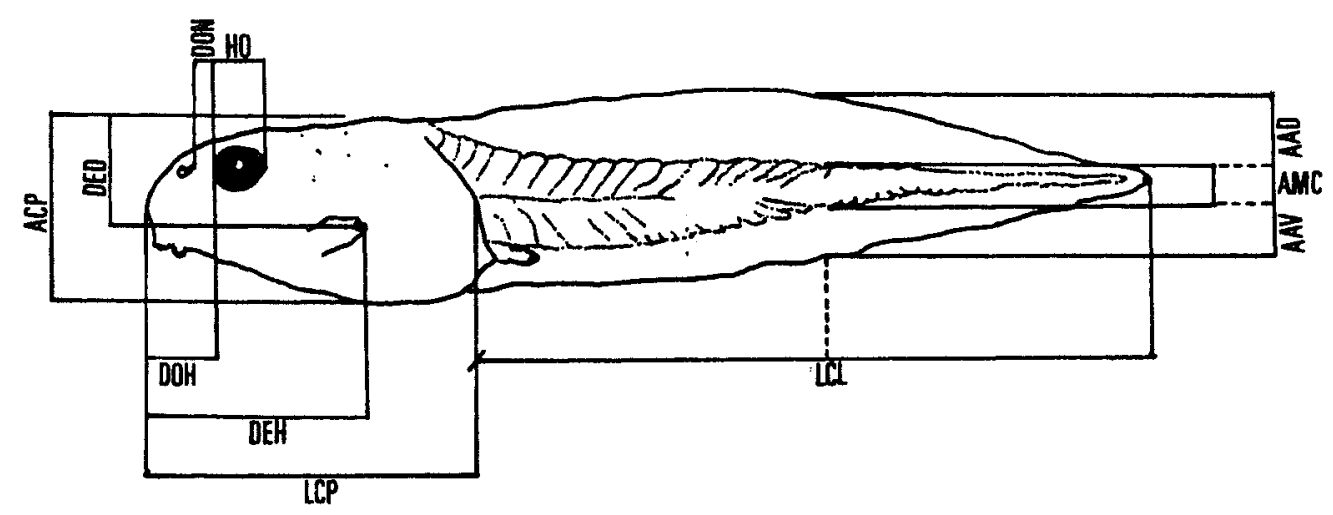

Fig. 1. Cuerpo completo, en vista lateral, con las estructuras larvarias más importantes y las diferentes medidas empleadas en la caracterización morfológica de las larvas de anuros. Las abreviaciones son como sigue: longitud cu erpo (LCP), longitud cola (LCL), altura cuerpo (ACP), distancia ojo-narina (DON), distancia ojo-punta hocico (DOH), diámetro ocular (HO), distancia espiráculopunta hocico (DEH), distancia espiráculo-dorso (DED), anch o musculatura caudal (AMC), altura al eta dorsal (AAD), altura al eta ventral (AAV). 

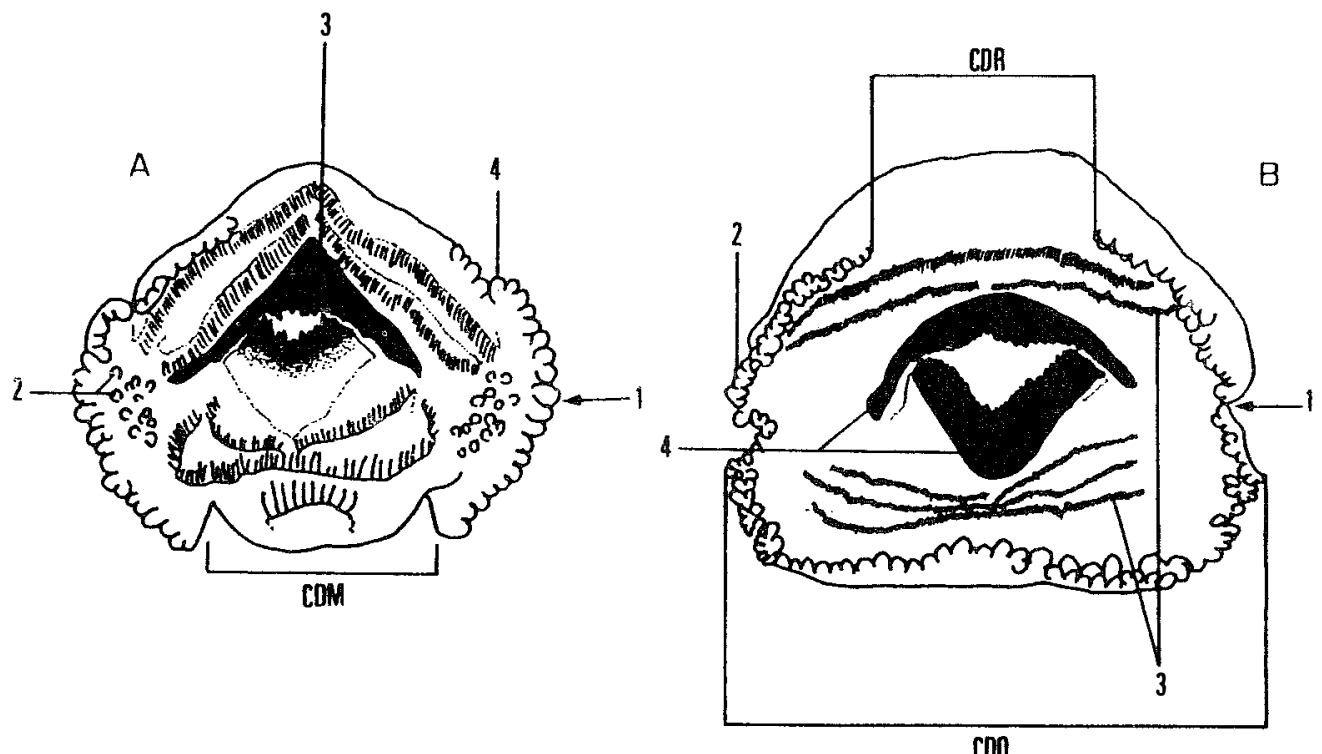

Fig. 2. Aparato oral de (A) Scinax grupo rostrata, en el que se identifican las partes más importantes que lo componen, designadas con un número o código, que se explican como sigue: ancho diastema mental (CDM), (1) disco transangular, (2) papilas intramarginales. (3) rostrodonte con sierras grandes agudas, (4) papilas marginales grandes, romas y en fila sencilla; (B) Nephelobates: ancho disco oral (CDO), ancho diastema rostral (CDR), (1) inflexión intramarginal, (2) papilas marginales en filas dobles, (3) filas de queratodontes, fórmula 2(2)/3(1), (4) rostrodontes con sierras pequeñas mixtas, más una inflexión media en el suprarostrodonte. Ambas Figs. no son necesariamente proporcionales entre sí.

Como recomendación final, debe tomarse en cuenta que, la plasticidad fenotípica de los renacuajos los hace organismos difíciles para ser categorizados en una clave. Es relativamente común encontrar una considerable variación individual, geográfica y ontogenética. También son frecuentes las alteraciones originadas por factores medioambientales; las anomalías del disco oral y sus componentes son igualmente comunes. Hay quetener presente que en cualquier grupo hay individuos que representan extremos extraordinarios que no se acomodan adecuadamente a las claves, y los renacuajos no son la excepción. Dentro de esta categoría se incluyen los ejemplares mal preservados (deshidratados o descompuestos), deformes o mutilados. 


\section{RESULTADOS}

\section{CARACTERES MORFOLÓGICOS}

\section{I.- Cuerpo}

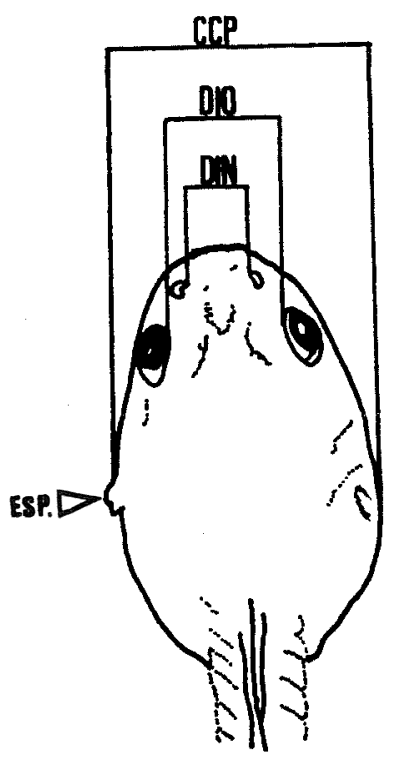

A

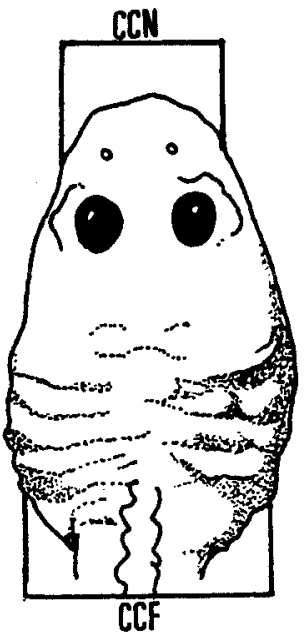

B

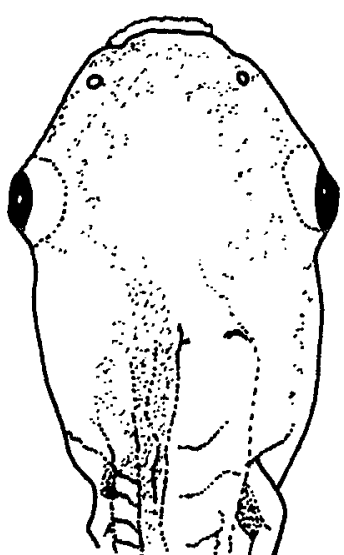

C

Fig. 3. Cuerpo en vista dorsal de (A) Hyla, (B) Centrolene, y (C) Scinax, en los que se observa, comparativamente, los ojos situados dorsolaterales, dorsales y laterales, respectivamente. En Hyla se observa al espiráculo en forma de un tubo libre corto (indicado por la flecha), ausente en los otros dos ejemplos. Se muestran las estructuras más importantes y las diferentes medidas empleadas en la caracterización morfológica de las larvas de anuros. Las abreviaciones son como sigue: ancho cuerpo (CCP), ancho del cuerpo a nivel de las narinas $(\mathrm{CCN})$, ancho del cuerpo al final del cuerpo (CCF, longitud más o menos equivalente a la distancia narinapunta hocico), distancia internarinal (DIN), distancia interocular (DIO). Las proporciones entre estas tres Figs. no necesariamente son las mismas. 


\section{1.- Configuración relativa del cuerpo en virtud de la proporción altura/ancho (altura del cuerpo sobre el ancho del cuerpo [acp/ccp]), medido a nivel del espiráculo (Figs. 1 y 3):}

(a) deprimido-ovalado (acp/ccp menor de 0.95)

(c) comprimido (acp/ccp mayor de 1.05)

(b) globular (acp/ccp entre 0.95 y 1.05)

\section{2.- Configuración del cuerpo en vista dorsal} (Fig. 3):

(a) elongado postero-triangular (cuerpo alargado [mucho más largo que ancho] con el extremo posterior menos ancho que el extremo anterior $[\mathrm{ccn}>\mathrm{ccf}])$

(b) elongado - ovalado (cuerpo alargado con los extremos posterior y anterior del cuerpo más o menos de igual de ancho $[\mathrm{ccn}=\mathrm{ccf}]$ )

(c) redondo - ovalado (en forma de huevo, igual que [b] excepto que el cuerpo es tan largo como ancho o apenas ligeramente más largo que ancho)

(d) cuneiforme (extremo anterior mucho menos ancho que el extremo posterior, en forma de cuña [ccf $>\mathrm{ccn}]$ )

(e) sinuoso (independientemente de su longitud, cuerpo sinuoso, con inflexiones laterales simétric as naturales)

3.- Elementos condrocraniales: Estructuras morfológicas internas de tejido blando o cartilaginoso (a veces osificadas [La Marca 986]) que pueden observarse, o no, a través de la piel del dorso de la cabeza, condición que parece tener un alto valor taxonómico (Sokol 1975).
(a) visibles
(b) invisibles

\section{II.- Ojos}

4.- Localización: Para ayudar a la localización de los ojos en casos ambiguos o algo confusos, se debe tratar de definir la dirección de enfoque de la pupila de la larva (en preservativo esta se torna de un color blanquecino opaco, ver Fig. 3).
(a) Dorsales
(c) laterales

(b) dorsolaterales

5.- Distanciainterorbital: El borde intraocular o margen medio, margen más interno entre cada "órbita" (señalada por el límite entre la piel dorsal y el comienzo del epitelio ocular transparente) y no entre las "globos oculares"(McDiarmid\& Altig 1989-1990: 52), se toma como referencia para medir la distancia entre ambos ojos. En el caso de la distancia internarinal se aplica igual criterio (margen medio entre cada narina (ver Lavilla\& Scrocchi 1986). Ambas distancias se comparan (ver Fig. 3).
(a) > distancia internarinal
(b) $<$ distancia internarinal

\section{III.- Narinas}

6.- Dirección de abertura: Para ayudar en la definición de la dirección de las narinas, se toman en cuenta solo la(s) posición(es) en qué se pueda observa el conducto narinal; en el caso en que sea desde una o tres direcciones se debe especificar en cuales direcciones se observa el conducto narinal (paracomentarios adicionales ver Van Dijk 1966, Lavilla 1988: Fig. 3). 

(a) lateral (d) parcialmente en dos direcciones
(b) dorsal (e) parcialmente en las tres direcciones

(c) anterior

\section{7.- $\quad$ Forma de la abertura (Fig. 4):}

a) Redondo-ovalada

b) Reniforme

c) Semicírculo (forma de "D")
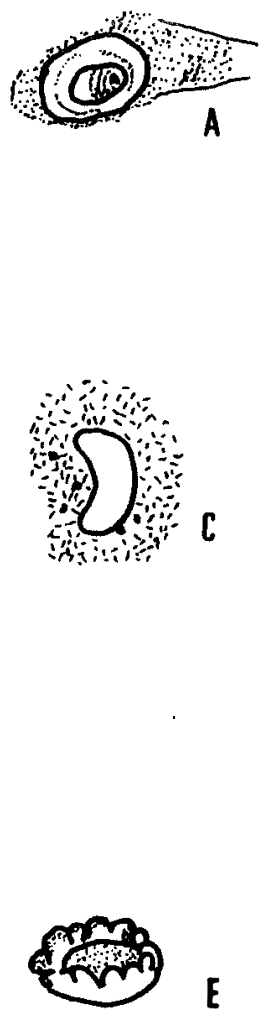

8.- Configuración del margen de laabertura narinal:Enél margen dela abertura narinal a veces se encuentra una carnosidad, generalmente incolora o ligeramente manchada y translúcida, denominada anillo marginal de la narina, si esta se proyecta por encima de la superficie del borde de la narina se denomina "Protuberante" (Ver Fig. 4. Comentarios adicionalesen Johnston \& Altig 1986, Lavilla 1988).

(a) presente-protuberante

(b) presente-no protuberante

(c) anillo ausente
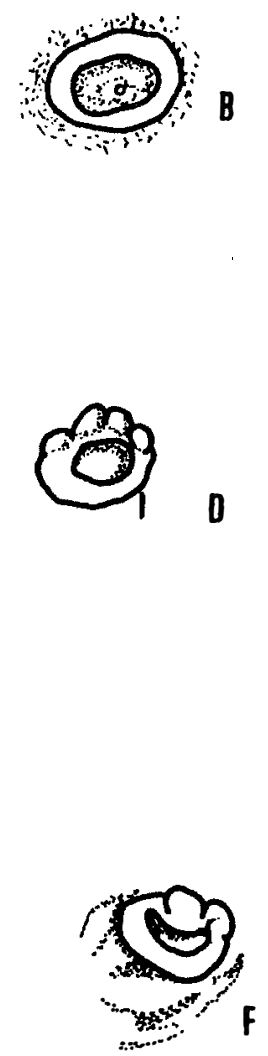

Fig. 4. Algunas configuraciones de la abertura narinal de los renacuajos de los altos Andes Venezolanos: (A) Nephelobates, (B) Mannophryne, (C) Hyla (grupo boans), (D) Colostethus leopardalis, (E) Hyla platydactyla, (F) Hyla jahni (E y F son del grupo bogotensis). Todas se tomaron en vista dorsol ateral, excepto la Fig. (C), la cual fue hecha en vista dorsal; los tamaños de las distintas Figs. no necesariamente son proporcionales. 
9.- Configuración del borde del anillo marginal: Se han detectado diferentes formas o características particulares del bordedel anillo marginal de la narina. En el caso de un borde "irregular" describe la ausencia de estructuras papiliformes claramente identificables, pero si un borde con el contorno sinuoso o irregular, que debe describirse en cada caso (otros ejemplos se mencionan en Channing 1984 , Channing et al. 1988, y Johnston \& Altig 1986). Ver Fig. 4.

(a) papiloso (desde una sola papila en adelante)
(b) irregular
(c) liso
(d) no aplicable (cuando el anilloes ausente)

10.- Posiciónanteroposterior: Deben medirse las siguientes distancias: borde posterior de la narina-borde anterior del ojo (DNO), y extremo del hocico-borde anterior del ojo (DHO). Finalmente secalcula la proporción DNO/DHO (para comentarios adicionales ver Van Dijk 1966, Lavilla \& Scrocchi 1986).

(a) más cerca del ojo $(\mathrm{DNO} / \mathrm{DHO}<0.45)$

(b) más cerca de la punta del hocico $(\mathrm{DNO} /$ $\mathrm{DHO}>0.55$ )

(c) equidistante (DNO/DHO entre $0.45 \mathrm{y}$ $0.55)$

\section{IV.- Espiráculo}

11.- Posición transversal: Altig (1970) define los siguientes conceptos como sigue: Mediógiro $=$ espiráculo situado en medio del vientre, Levógiro= espiráculo situado del lado izquierdo del cuerpo. Sin embargo, este último concepto es poco empleado en la literatura, siendo más común el término "siniestro" (ver Figs. 1 y 3 ).
a) mediógiro
b) siniestro (=levógiro)

12.- Posición anteroposterior: Se determina midiendo la longitud desde la punta del hocico hasta el extremo más distal del espiráculo y esta medida se contrasta porcentualmente con la longitud corpórea. La anotación de la posición se hará considerando si el espiráculo se encuentra antes, sobre o posterior al punto medio del largo del cuerpo (lcp) medido desde la punta del hocico (Fig. 1).

(a) anterior (< del $45 \%$ del lcp)

(b) medio (entre $45 \%$ y $55 \%$, ambos inclusive, del Icp)

(c) posterior ( $>$ del $55 \%$ del lcp)

13.- Posición dorsoventral: Se determina midiendo la longitud desde el dorso hasta aproximadamente la mitad de la abertura espiracular. La anotación se hará considerando si la medida resultante se encuentra por arriba, sobre o por debajo del punto medio de la altura del cuerpo (acp) medido desde el dorso (Fig. 1).
(a) arriba $(<$ del $45 \%$ del acp)
(b) medio (entre $45 \%$ y $55 \%$, ambos inclusive, del acep)

(c) abajo (> del 55\% del acp) 
14.- Tubo espiracular externo: Cuando los pliegues dérmicos del espiráculose separan totalmente del cuerpo extendiéndose formando un verdadero tubo libre (ver Fig. 3). Para ejemplos extremos, ver Wassersug \& Pyburn (1987). Está ausente cuando no hay una estructura tubular claramente separada del cuerpo, inclusive si sólo la mitad del tubo está fusionado lateralmente al cuerpo (Fig. 3B-C).

(a) Presente (formando un verdadero tubo libre)

(b) Ausente

15.- Dirección de la abertura espiracular: Aquí se intenta describir la dirección en que está orientado el tubo espiracular (y cuando es necesario, también la abertura del espiráculo) respecto a la línea media longitudinal del cuerpo (explicación más extensa en Van Dijk 1966).

a) dorsal (dirigido franco hacia el dorso)

b) dorsoposterior (dirigido oblicuamente entre el dorso y el extremo posterior del cuerpo)

c) posterior (dirigido franco haciael extremo posterior del cuerpo)

d) posterolateralmente (dirigido hacia el extremo posterior del cuerpo pero con la abertura perfectamente visiblelateralmente)

\section{V.- Cloaca}

La cloaca o abertura cloacal, no debe ser denominada "ano"o "abertura anal", etc., debido que la estructura observada en los renacuajos no es equivalente a la que se observa en otros verte- brados superiores. (también ver Van Dijk 1966). Normalmente siempre hay pliegues carnosos alrededor o relacionados con la cloaca que a pesar de no formar una estructura tubular separada del cuerpo (o cola), se denominará en adelante, por conveniencia, tubo cloacal.

16.- Posición anteroposterior: Según la propuesta de Van Dijk (1966) está característica está relacionada con la vinculación o no de la cloaca con la cola (generalmente las aletas caudales, Fig. 5).

(a) caudal (si hay algún vínculo con la cola)

(b) abdominal (si no hay ningún vínculo con la cola)

17.- Configuración del tubo cloacal: Lateromarginal define al tubo cloacal formado por un pliegue tubular de la aleta ventral que se extiende lateralmente a esta; mediomarginal es el tubo cloacal en posición media que se extiende por el margen inferior de la aleta ventral; el tubo cloacal mediolineal es uno muy similar al anterior excepto a que se extiende por el margen inferior de la musculatura caudal, en aparente desvinculación con la aleta ventral, originándose ésta donde el tubo cloacal termina (según la modificación hecha a la propuesta de Van Dijk 1966; ver Fig. 5).
(a) lateromarginal
(b) mediomarginal
(c) mediolineal
(d) extracaudal (cloaca abdominal) 

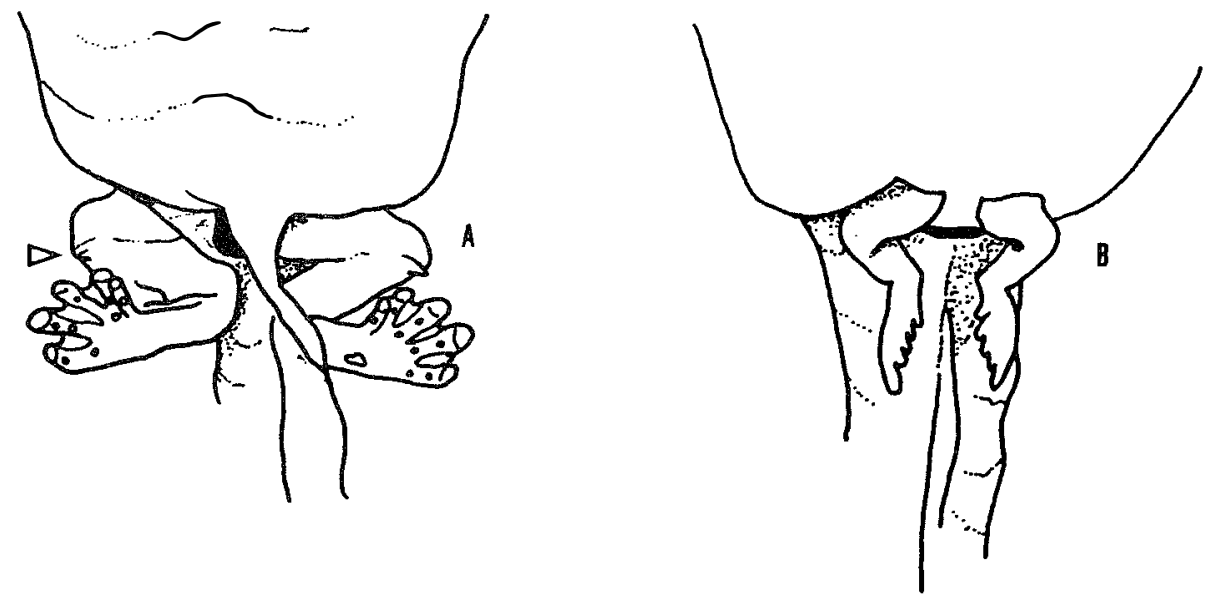

Fig. 5. Detalle esquemático en vista ventral de la localización de la cloaca, (A) abdominal (Hyla grupo labialis), (B) caudal media (Atelopus). La flecha indica el pliegue dérmico a nivel de la coyuntura tibio-femoral, en los miembros traseros en desarrollo.

18.- Posición transversal: Esta posición se define con base enla dirección en la que esté dirigida la abertura cloacal, independientemente del lugar de origen del tubo cloacal en el cuerpo; es decir, si el tubo cloacal se origina del lado izquierdo del cuerpo pero la abertura se sitúa del lado derecho del cuerpo, se registrará la posición como diestra (Johnston \& Altig 1986). Como punto de referencia longitudinal para la determinación de los lados derecho e izquierdo se tomará la aleta caudal ventral. Las posiciones hasta hoy registradas son:

(a) diestra (ladoderecho de la a leta ventral)

(b) media (alineada con la aleta ventral)

\section{VI.- Cola}

Se entiende que la cola esta comprendida por la aleta caudal (la cual es una única estructura que, por conveniencia, es dividida en dos porciones, la dorsal y la ventral, tomándose como punto de divergencia, el vértice distal [Van Dijk 1966]), y la musculatura caudal.

\section{9.- Longitud de la musculatura caudal en} relación con la longitud total de la cola: El extremo final de la cola está definida por ser el punto más distante de la cola sea este aleta caudal (AC) o musculatura caudal (MC) o ambos sean de igual longitud.

(a) igual (longitud $\mathrm{AC}=\mathrm{MC}$ )

(b) menor (longitud $\mathrm{MC}<\mathrm{AC}$ ) 

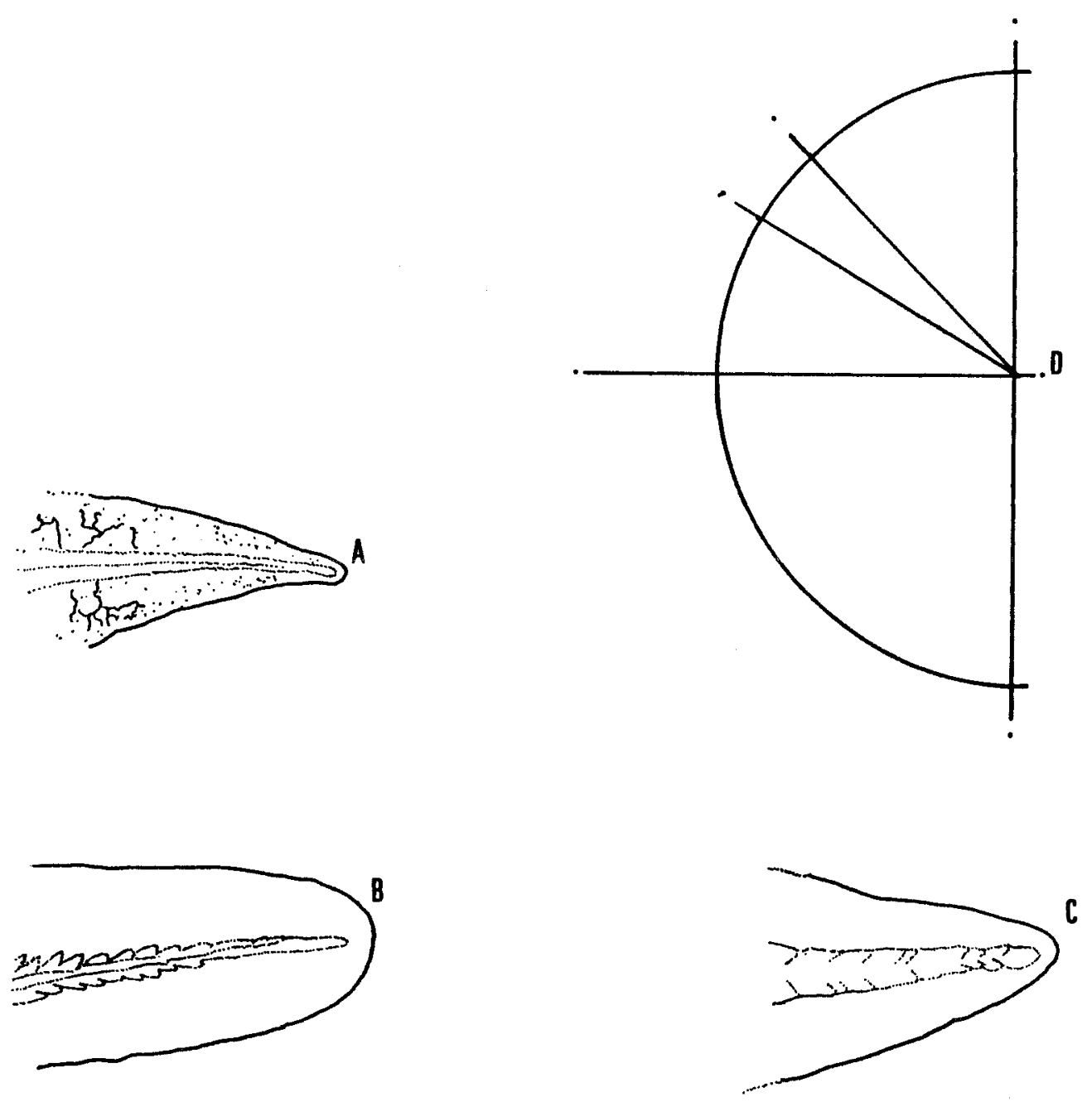

Fig. 6. Configuración del extremo distal de la cola, (A) agudo (Scinax), (B) redondeado (Atelopus), y (C) subagudo (Hyla grupo labialis), incluyendo un ejemplo de marco de referencia angular (D), para facilitar la determinación del ángulo formado por el borde de la aleta caudal. Los tamaños de los extremos caudales no guardan ninguna proporción entre si.

\section{0.- Configuración delextremodistal caudal:}

En este apartado se sigue una calificación relativamente arbitraria, modificando la técnica propuesta por Van Dijk (1966). Para medir los ángulos, la Fig. 6D sirve como ejemplo: la línea horizontal se hace coincidir con el eje longitudinal de la cola y se coloca el extremo distal de la cola en el puntode convergencia de la línea horizontal con el segmentoperpendicular, procediendo a determinar si la tangente que pasa por el margen libre de la aleta dorsal es menor, igual o mayor de $30^{\circ} 045^{\circ}$ según sea el caso (Fig. 6). 

(a) redondeado (ángulo mayor de $45^{\circ}$ )
(b) subagudo (ángulo entre $30^{\circ}$ y $45^{\circ}$ )
(c) agudo (ángulo menor de $30^{\circ}$ )
(d) filiforme (en forma de filamento estrecho)

21.- Miómeros o miotomos: Unidades musculares de la cola, divididos a lo largo del centro de la cola. A veces el tamaño y extensión del "tomo muscular" (unidades musculares diferenciables) es comparativamente muy útil para definir las costumbres torrentícolas de algunos renacuajos.
(a) indistinguibles
(b) visibles en 1/1 de la cola
(c) visible parcial o totalmente a lo largo del $1 / 2$ proximal de la cola
(d) visible parcial o totalmente a lo largo del 1/2 distal de la cola

22.- Eje longitudinal de la cola: La línea formada en el punto de unión de los miómeros superiores e inferiores a todo lo largo de la musculatura caudal se toma como eje longitudinal de la cola (comentarios adicionales en Van Dijk 1966). Ver Figuras 1 y 6.
(a) dobla hacia abajo posteriormente
(b) recto

23.- Punto de origen y anchura de las aletas caudales: La anotación que se presenta abajo se refiere al punto donde se origina la aleta y la proporción de anchura de la misma (punto de origen / ancho). Las medidas de las porciones dorsal y ventral de las aletas caudales se toma a la mitad de la longitud total de la cola, respecto al ancho de la musculatura caudalen el mismo punto (Fig. 1). La anotación debe hacerse tanto para la porción dorsal como para la porción ventral, respectivamente.
(a) sobre cola / menor
(b) sobre cola / igual
(c) unión cuerpo-cola / menor
(d) unión cuerpo-cola / igual

(e) unión cuerpo-cola / mayor

(f) sobre cuerpo / menor

(g) sobre cuerpo / igual

(h) sobre cuerpo / mayor

\section{VII.- Patas Traseras}

24.- Punto de origen de las patas traseras: Esta caracter se toma considerando la ubicación de las yemas de los miembros traseros respecto al tubo o abertura cloacal (modificado de la propuesta según Van Dijk 1966).

(a) supracloacal

(b) intra-paralelo (desarrollo de las patas al mismo nivel que el tubo cloacal y con al menos uno de los miembros dentro del tubo cloacal) 
(c) extra-paralelo(igual que anterior excepto que ningún miembro está dentro del tubo cloacal)

25.- Configuración patas traseras: Se refiere a la presencia o no de pliegues dérmicos, más comúnmente en las rodillas y/o en los talones (más detalles ver Van Dijk 1966). Ver Fig. 5.
(a) con pliegues dérmicos (indicandodonde)
(b) sin pliegues dérmicos

\section{VIII.- Aparato Oral}

El aparato oral está constituido por la "boca" (indicada por los "queratostomas", ver abajo) y, generalmente, una estructura carnosaen formade disco sobresaliente que rodea a la boca (Van Dijk 1966). Ver Fig. 2.

\section{6.- Tipos de aparato oral:}

(a) simple (disco oral apenas desarrolladoo ausente y sin partes orales queratinizadas)

(b) normal (disco oral presente + partes orales queratinizadas, sin variación apreciable en la configuración de estas partes durante los estadios prometamórficos)

(c) modificable (aparato oral presente + partes orales queratinizadas en estadios premetamórficos, con importante variación en la configuración de estas últimas entre los estadios premetamórficos y prometamórficos)

(d) complejo (disco oral normal + disco succionador abdominal adyacente al disco oral)

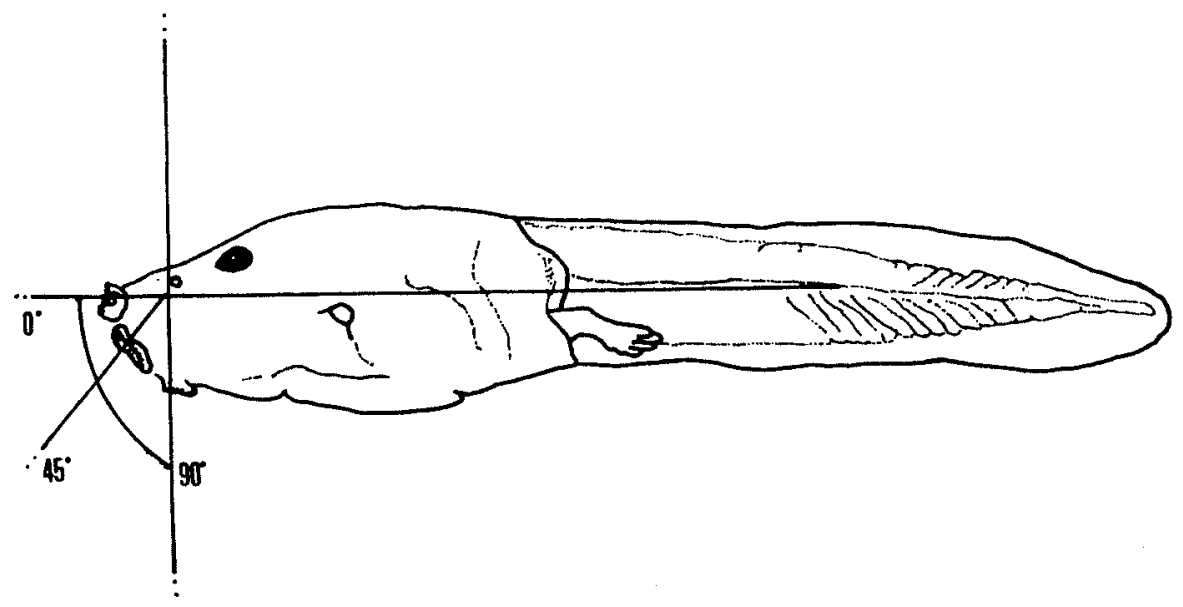

Fig. 7. Localización del aparato oral en relación con su posición en el cuerpo: anterior $\left(0^{\circ}\right)$, anteroventral $\left(45^{\circ}\right)$, ventral $\left(90^{\circ}\right)$, respecto al eje longitudinal del cuerpo. 
27.- Posición en el cuerpo: La posición de disco oral en el cuerpo reviste una importancia vital, $n$ osolocomo caracter de valor taxonómico, sino por su significado adaptativo ecomorfológico (Altig \& Johnston 1989). Estos últimos autores han propuesto un método para calcular con precisión la posición correcta del disco oral en el cuerpo. Este se ha modificado con el propósito de facilitar su aplicación de la manera siguiente: el ángu lo formado por el aparato oral respecto al eje longitudinal del cuerpo determinará su posición relativa; para lograr esto, se intentará definir la "dirección" del aparato oral (bastaría con ubicar los queratostomas como punto de referencia), ver Fig. 7), y se "mide" el ángulo aproximado respecto a la recta imaginaria del eje longitudinal del cuerpo. Debido a que, probablemente, sólo se obtendrán medidas aproximadas, se emplearan únicamente tres "medidas extremas" (ME), $0^{\circ}$ (eje longitudinal del cuerpo), $45^{\circ}$, y $90^{\circ}$ (recta imaginaria perpendicular al e je long. del cuerpo), que representan las posiciones "anterior", "anteroposterior", y "ventral", respectivamente; los cálculos intermedios entre estas "medidas extremas", se aproximaran hasta la ME más próxima
(a) ventral (b) anteroventral
(c) anterior

28.- Tamaño del disco oral: Medidas hechas en la parte de máxima anchura del disco oral (Fig. 2), respecto a la anchura del cuerpo(ccp, estaúltimamedidahechacomo se explica en el numeral 1). Para el caso particular del tipo de aparato oral "Complejo" (ver Apartado 27, numeral d), solo se tomará el ancho del disco oral, no del disco succionador abdominal.

(a) muy pequeño ( $\leq 20 \%$ del ccp) (b) moderadamente pequeño ( $>20 \%$ hasta $\leq 40 \%$ del ccp)

(d) grande ( $>60 \%$ del ccp)

(c) mediano ( $>40 \%$ hasta $\leq 60 \%$ del ccp)

(e) no aplicable (disco oral ausente)

29.- Configuración del disco oral: Se refiere a la presencia o no de inflexiones laterales del disco oral (ver Lavilla 1988); el disco oral se divide en supraangular e infraangular dependiendo a si se hace referencia a la parte del disco oral situada por encima o debajo del ángulo formado por los queratostomas; es decir, dirigida hacia el extremo anterior del hocico o hacia el vientre, respectivamente (Van Dijk 1966). Ver Fig. 2.

(a) transangular (sin inflexiones laterales)

(b) intraangular (con inflexiones laterales)

\section{IX.- Papilas Orales}

Estas estructuras orales carnosas, presentes en los bordes o dentro del disco oral, dan una apariencia "dentada" al disco oral y con mucha frecuencia fueron erróneamente descritas como "indentación lateral", ya que no tienen ninguna relación con las estructuras ora les queratinizadas dentiformes (Mijares-Urrutia 1991). Ver Fig. 2.

30.- Extensión de las papilas orales: En este apartadosedebenaclararun parde términos; las papilas marginales están con una gran frecuencia divididas por "brechas" (mejor conocidos como "diastemas", espacios en que la(s) línea(s) de papilas marginales se interrumpen o desaparecen) que, por su ubicación, se denominan "rostral" y "mental", según estén presentes en los 
extremos de las porciones supraangular o infraangular del disco oral, respectivamente (ver también Lavilla 1988). Ver Fig. 2.

(a) circumoral (completas alrededor del disco oral)

(b) separadas por diastema rostral

(c) separadas por diastema mental

(d) separadas por diastemas rostral y mental

\section{1.- Configuración de las papilas orales} marginales: Van Dijk (1966), of rece esta lista pero sin detallar cada uno de los distintos aspectos. Una "fila sencilla alternada" identifica a una sola secuencia de papilas pero, cada papila está situada oblicuamente respecto a la vecina (en zigzag) en el plano longitudinaldel margen del disco oral. "Fila sencilla alineada" es similar a la anterior excepto en que las papilas no están en zigzag sino alineadas a el mismo plano longitudinal del margen del disco oral. "Fila doble" describe el caso cuando dos papilas se sitúan en el mismo plano perpendicular a ambos lados del margen del disco oral; cuando tres o más papilas se encuentran en el mismo plano perpendiculardel margendeldiscooralnos encontramos con la condición "filas múltiples". El término "mixtas" se aplica en los casos en que se combinen dos de las características descritas, sean ambas de configuración (alineadas y alternadas) o cantidad (sencillas y dobles) y dadoel caso, se debe mencionar la porción del disco oral donde se presenta cada una de dichas caractarísticas (Fig. 2).
(a) fila sencilla alternada
(b) fila sencilla alineada
(c) filas en configuración mixta
(d) filas dobles
(e) filas en cantidad mixta
(f) filas múltiples

32.- Tipos de papilas orales marginales: Mientras más ancha es la base de la papila esta tiende a ser más corta y, a la inversa, si la base es estrecha la papila tiende a ser larga.Estocreaunaseriede combinaciones de tipos de papilas que tiene un apreciable valor taxonómico. Dependiendo de las características de la forma y tamaño del extremodistal de las papilas marginales, se han identificado seis combinaciones diferentes. En los casos de papilas con tamaño o forma mixta, se debe indicar la porción del disco oral en que se ubica cada característica.
(a) papilas ausentes
(b) largas y agudas
(c) largas y romas
(d) largas con extremo mixto
(e) cortas y romas
(f) mixtas de tamaño

33.- Papilas intramarginales: Papilas de iguales características que las marginales pero distribuidas dentro (intramarginalmente)deldisco oral que, en los grupos estudiados se presentan por lo común lateralmente.
(a) presentes lateralmente
(b) ausentes 


\section{X.- Queratostomas}

Estas estructuras, en general mal llamadas "picos", debido a que este término tiene ciertas implicaciones morfológicas no aplicables en el caso de los renacuajos, e igualmente el término "rostrodontes" (más o menos $=$ dientes de la cara), por tener un significado que evidentemente es inadecuado respecto a la estructura que pretende describir. Se propone el empleo de la palabra "Queratostomas" (más o menos, "boca queratinizada"), por considerar que hace referencia más precisa de la estructura tratada. Los queratostomas se encuentran en medio del disco oral y es, por llamarlo de una forma más comprensible, y en armonía con la propuesta anterior, la "boca" del renacuajo. Varía de tamaño y forma, y puede o no presentar queratinización, y cuando presente, esta puede exhibir diversos grados de intensidad. Ver Fig. 2.

\section{4.- Constitución de los queratostomas: Los} queratostomas desde su parte basal hasta su extremo libre pueden presentar diferente densidad de queratinización, o inclusive no estar queratinizados. En tal caso, generalmente el queratostoma se encuentra cornificado o "endurecido", más o menos como una uña. Esto último puede variar dependiendo del estadio de desarrollo larvario o ser característico de la especie a lo largo de toda su etapa larvaria.

(a) completamente queratinizado $(100 \%$ queratinizado o al menos casi)

(b) parcialmente queratinizado (parte basal sin queratina, apareciendo e intensificándose esta gradualmente hacia el margen libre)

(c) sin queratina peroendurecidos (córneos o calloso)
35.- Forma del supraqueratostoma: Básicamente aquí se hace referencia a la forma que adquiere la parte queratinizada del queratostoma como un todo (desde su base hasta el margen libre), tomando en cuenta su amplitud o estrechez.
(a) "A" (en arco)
(b) "M"

c) " $\Pi$ " (cuadrangular, procesos laterales + porción media en ángulo recto)

36.- Forma del infraqueratostoma: Ver descripción en el apartado 35.
(a) "V" ancha
(b) "U" ancha
(c) "A" estrecha

37.- Configuración del margen libre de los queratostomas: El margen libre de los queratostomas presenta normalmente unas irregularidades relativamente constantes en tamaño, forma y distribución, denominadas "sierras". Las sierras de los queratostomas pueden variar, dependiendo de la especie o grupo de especies, en tamañoy forma según los diferentes estadios de desarrollo. Es recomendable que, en general, se registren las características de estos con renacuajos en estadios entre 32 y 39 de desarrollo para asegurar una adecuada descripción. El tamaño de las sierras puede tratar de definirse comparándolo con el de los queratodontes en relación con si son menores, iguales o mayores en tamaño que estos últimos; cuando son muy pequeños, inclusive en los casos extremos en que debe confirmarse bajo la lupa estereoscópica con una alta magnificasión (alrededor de $25 x$ ) estosse denominan como "diminutas". En ocasiones se pueden observar sierras de tamaño variable y/o aisladas o grupos de sierras separadas por espacios lisos, si esta 
condición se observa en la mayoría de las larvas examinadas de la especie, puede que esta sea la situación natural que se ha denominado "irregular o crenulado". En el caso en que el queratostoma no exista o no este queratinizadoeste apartado nose aplica.
(a) grandes y agudas
(b) pequeñas mixtas (agudas + romas)
(c) pequeñas y romas
(d) diminutas
(e) ausentes (lisos)
(f) irregular o crenulado

(g) no aplicable

\section{8.- Otras características del margen libre} de los queratostomas: Este apartado se refiere a las características resaltantes que modifican el borde distal de los queratostomas, distintos e independientemente de la presencia del sistema de "sierras". Estos son proyecciones o inflexiones del queratostoma de támaño y forma (normalmente mayores que las sierras) y ubicación definidas (Fig. 2).

(a) sin inflexiones o proyecciones

(b) supraqueratostoma con una muesca media

\section{XI.- Queratodontes}

Se toma este nombre y su definición (Van Dijk 1966) por ser la que más se adecua a las características morfológicas de esta estructura oral. Los queratodontes son estructuras "dentiformes", constituidos por queratina y se originandeunafunda("sheath") o pliegue carnoso que los sostiene. Se presentan en filas que pueden ser continuas o discontinuas. Son estructuras frágiles que pueden desprenderse por manipulación brusca o "desaparecer" total o parcialmente en el preservativo o cuandoel animal es criado en cautiverio. Sin embargo, el pliegue carnoso que les sirve de base siempre indica fielmente la longitud y la continuidad de la fila. Puede ser una característica muy variable según el estadio de desarrollo(pore jemplo las larvas del grupo de Hyla bogotensis) o ser muy estable a lo largo del desarrollo desde estadios muy jóvenes (estadio 23-25) hasta los prometamórficos avanzados (estadio 39), variando solo el tamaño de los queratodontes individuales (por ejemplo como en: Colostethus, Mannophryne, y Nephelobates). Ver Fig. 2.

39.- Fórmula de filas de queratodontes: $\mathrm{La}$ anotación de esta característica se hace según las recomendaciones de Altig (1970) y Altig \& Johnston (1986), modificados según el sistema propuesto por Dubois (1994). Cuando se determineque noexistan queratodontes este apartado no se aplica y se debe indicar como "Queratodontes ausentes"..

(a) no aplicable

(b) $1 / 2$

(c) $2\left[\mathrm{~A}_{2}\right] / 2\left[\mathrm{P}_{1}\right]$

(d) $2 / 3$

(e) $2\left[\mathrm{~A}_{2}\right] / 3$

(f) $2\left[\mathrm{~A}_{2}\right] / 3\left[\mathrm{P}_{1}\right]$

(g) $2 / 3\left[P_{1}\right]$

(h) $2\left[\mathrm{~A}_{2}\right] / 4\left[\mathrm{P}_{1}\right]$

(i) $4\left[\mathrm{~A}_{4}\right] / 6\left[\mathrm{P}_{1}\right]$ 
(j) $6-8\left[\mathrm{~A}_{6}-\mathrm{A}_{6}\right] / 8-11\left[\mathrm{P}_{1}\right]$

(k) $11-14\left[\mathrm{~A}_{11}-\mathrm{A}_{14}\right] / 12-15\left[\mathrm{P}_{1}\right]$

40.- Longitudfilasdequeratodontes: Las filas de queratodontes por debajo del infraqueratostoma se denominan queratodontes infraangulares (=QIA), y las filas que se encuentren por sobre el supraqueratostoma, ' queratodontes supraangulares $(=Q S A)$ (Lavilla 1988; Van Dijk 1966). Se comparan aquí las longitudes respectivas de las filas infra y supraangulares, tomando como referencia la(s) fila(s) más larga(s) a cada lado de los rostrodontes.
(a) QIA > QSA
(b) $\mathrm{QIA}=\mathrm{QSA}$
(c) QIA $<$ QSA

(d) no aplicable (queratodontes ausentes)

41.- Configuración de las filas de queratodontes (Fig. 2). Normalmente las filas suprae infraangulares dequeratodontes se observan formando una arco alrededor del queratostoma, o siguiendo la silueta del disco oral; por esta última razón, algunas veces ocurre que la(s) fila(s) forma(n) un "ángulo" cerrado fuera de la zona media del labio, pero sin quebrarse o separarse (es decir, el pliegue carnoso que los sostiene aun es continuo) o desaparecieren los queratodontes por lo que la anotación debe hacerse como "angulosa". De no existir ni los queratodontes ni el pliegue carnoso que indica que alguna vez existieron entonces este apartado no se aplica.

(a) en semicírculo o en arco sinuoso

(b) angulosas

(c) no aplicable

\section{CLAVES}

Claves para la identificación de las familias y géneros de las larvas de anuros que habitan los altos Andes venezolanos. Cuando para una determinada familia y/o género sólo se conozca una especie o se halla descrito el renacuajo de una sóla especie, esteseráindicadoen la clave. Muchas características se mencionan aquí de manera incompleta puesto que se sobreentiende que han sido descritas con suficiente detalle en la parte anterior.

A 1.- Partes orales queratinizadas presentes a lo largo de todos o algunos de los estadios de desarrollo del renacuajo .... (B)

A2.- Partes orales queratinizadas ausentes a lo largo de todo el desarrollo larvario Hylidae (parte): Flectonotus

B l.- Papilas marginales orales separadas por, al menos, una diastema mental.

B2.- Papilas marginales orales circumorales o separadas solo por una diastema rostral

Cl (Bl).-Aparato oral comple jo (disco oral + dis co su ccionador abdominal), papilas marginales orales separadas solo por una diastema mental Bufonidae (parte): Atelopus

C2.- Aparato oral normal, papilas marginales orales separadas por diastemas rostral + mental

DI (C2).- Aletas caudales con origen sobre el cuerpo, más altas que la musculatura caudal, extremo distal caudal agudo o filiforme Hylidae (parte): Scinax grupo rostrata

D2.- Aletas caudales con origen sobre la cola o unión cuerpocola, iguales o menos altas que la musculatura caudal, ex tremo distal caudal redondeado Bufonidae (parte): Bufo

El (B2) - Fórmula de filas de queratodontes igual o menor a $2 / 2$

E2.- Fórmula de filas de queratodontes igual o mayor a 2/3

Fl (El).- Infraqueratostoma en forma de arco estre cho ("A"), papilas marginales orales en fila sencilla, cuerpo elongado redondo-ovalado en vista dorsal.

Centrolenidae (parte): Centrolene andinum 
F2.- Infraqueratostoma en forma de "U" ancha, papilas marginales orales en filas múltiples, cuerpo en vista dorsal cuneiforme

Hylidae (parte): Hyla grupo labialis

GI (E2).- Fórmula de filas de queratodontes $=2 / 3$

G2.- Fórmula de filas de queratodontes $>2 / 3$

Hylidae (parte): Hyla (J)

Hl (GI).- A bertura de la narina bordeado por anillo carnoso, coloración de la cola pardo oscuro o manchado

H2.- Abertura de la narina sin anillo carnoso, coloración de la cola entre blanco-crema inmaculado y crema sucio

11 (H2).- Espiráculo situado al final del cuerpo; abertura cloacal media Centrolenidae (parte): Hyalinobatrachium fleischmanni

12.- Espiráculo situado aproximadamente en la mitad longitudinal del cuerpo; abertura cloacal diestra

Leptodactylidae (Leptodactylus)

J I (G2) - Abertura de la narina reniforme, sin anillo marginal; fórmula máxima de filas de queratodontes $2 / 4$

Hylidae (parte): Hyla grupo boans $[\boldsymbol{H}$. crepitans]

J2.- A bertura de la narina redondo-ovalada con anillo marginal, fórmula mínima de filas de queratodontes $4 / 6$

.Hylidae (parte): Hyla grupo bogotensis

K I (HI).- Narina reniforme, extremo distal de la cola agudo, disco oral transangular ... Hylidae (parte): Hyla lanciformis
K2.- Narina redondo-ovalada, extremo distal de la cola subagudo, disco oral intraangular.

Dendrobatidae $(\mathrm{L})$

Ll (K2).- Filas de papilas marginales del disco oral mixtas (fila sencilla supraangularmente + filas dobles in fraangularmente), coloración de la cola uniforme mente pardo oscuro

Dendrobatidae (parte): Aromobates [nocturnus]

L2.- Filas de papilas marginales del disco oral en fila sencilla alrededor del disco oral, coloración caudal jaspeado de pardo sobre fondo crema o crema pardusco (M)

M I (L2).- Queratostomas con sierras grandes y agudas, anillo protuberante de la narina papiloso

Dendrobatidae (parte): Colostethus [C. leopardalis]

M2.- Queratostomas con sierras pequeñas agudas y/o roma $\mathrm{s}$ anillo protuberante de la narina liso

NI (M2).- Distancia internarinal menor que la distancia interorbital, parte media del margen libre del supraqueratostoma con sierras agudas grandes o con una concavidad sin sierras

Dendrobatidae (parte): Nephelobates

N2.- Distancia internarinal mayor que la distancia in terorbital, parte media del margen libre del supraqueratostoma con sierras agudas pequeñas o diminutas y sin concavidad

Dendrobatidae (parte): Mannophryne 


\section{DESCRIPCION DEL RENACUAJO DE Hyla pelidna Duellman, 1989}

Las larvas de $H$. pelidna, lote ULABG 3197 , está constituido por 59 ejemplares, en estadios que van desde el 25 hasta el 41 . Se recolectaron en la región del Páramo de Tamá, Distrito Páez, del Estado Apure, en Agosto de 1987, por E. La Marca, J.M. Rengifo, M. Sosa y J.E. GarcíaPérez, asociados con adultos recolectados en la misma localidad.
Algunas medidas del renacuajo de H. pelidna de los ejemplares en estadio $37(\mathrm{~N}=6)$, son como sigue [media \pm Desviación estándar (ámbito)]: Largo total 54.5 \pm 3.1 (51.3-59.0), largo cuerpo $18.2 \pm 0.8$ (17.2-19.7), ancho cuerpo $11.6 \pm 0.6$ (10.8-12.6), altura cuerpo $11.0 \pm 0.4$ (10.6-11.7), ancho aleta dorsal $4.1 \pm 0.5$ (3.3-4.7), ancho aleta ventral $4.0 \pm 0.5$ (3.0-4.7), distancia espiráculopunta del hocico $12.9 \pm 0.6$ (12.1-13.6), ancho del disco oral $4.1 \pm 0.3$ (3.8-4.7), distancia interorbital $7.1 \pm 0.4$ (6.8-7.8), distancia internarinal $5.7 \pm 0.4(5.0-6.4)$. En la Tabla 1 se of recen las medidas porcada estadio de desarrollo estudiado.
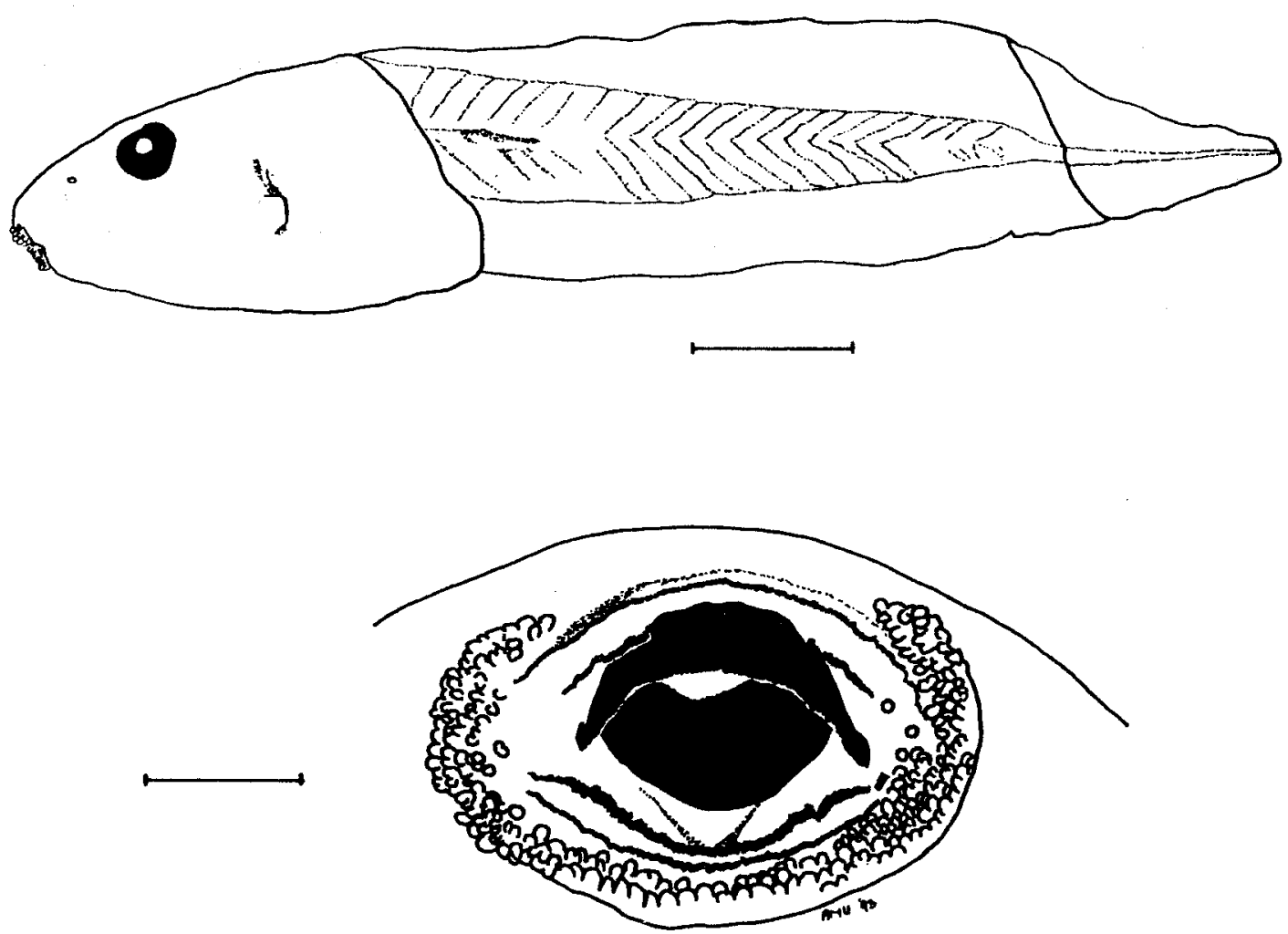

Fig. 8. Renacuajo de Hyla pelidna (ULABG 3197), (A) cuerpo en vista lateral, (B) aparato oral, en estadio de desarrollo 28. Escalas =(A) $5.0 \mathrm{~mm}$. (B) $1.0 \mathrm{~mm}$, respectivamente. 
Cuerpo deprimido-globular (apenas 14\% más anchoque alto), ovaladoen vistadorsal.Elementos condrocraniales invisibles dorsalmente. Ojos situados dorsolateral-lateralmente. Diámetro ocular alrededor del $36 \%$ de la distancia interorbital. Distancia interorbital mayor que la distancia internarinal (15\% mayor). Diámetro narinal casi $14 \%$ de la distancia internarinal. Narinas de forma redondo-ovalada, situadas entre el ojo y la punta del hocico ( $51 \%$ de la distancia oj -hocico desde el ojo), dirigidas anterolateralmente. Margen de la abertura narinal con anillo carnoso presente, no protuberante, liso. Espiráculo siniestro, formando un tubo visible lateralmente, totalmente adherido al cuerpo y dirigido dorsoposteriormente. Tubo espiracular situado a $75 \%$ de la longitud del cuerpo desde la punta del hocico, y 63\% de la altura del cuerpo desde el dorso. Abertura cloacal diestra, abdominal, no marginal, formando un tubo libre muy corto ( $8 \%$ de la longitud del cuerpo). Musculatura caudal alcanza el extremo distal de la cola. Extremo distal de la cola agudo (no filiforme), ligeramente curvado hacia arriba respecto al eje longitudinal de la cola. Miómeros visibles a todo lo largo de la musculatura caudal. Aletas caudales ligeramente más anchas que la musculatura caudal a la mitad de su longitud (aleta dorsal casi $20 \%$ más alta, aleta ventral $12 \%$ más alta). Aleta dorsal con origen en la unión cuerpo-cola. Aleta ventral con origen en el cuerpo (sobre extremo final del vientre). Los miembros posteriores se originan dorsalmente respecto al tubo cloacal, y presenta un pliegue transversal a nivel de la coyuntura tibio-femoral.

Disco oral situado anteroventralmente, de tamaño pequeño (30\% del ancho del cuerpo), transangular, bordeado por papilas marginales romas, dispuestas en filas múltiples y separadas por una escotadura rostral. Papilas intramarginales presentes lateral e infraangularmente. Queratostomas completamente queratinizados. Margen libre de ambos queratostomas sin inflexiones oproyecciones, con sierras diminutas, romas. Supraqueratostomas en forma de "A" ancha. Infraqueratostoma en forma de "U" ancha. Fórmula de filas de queratodontes $1 / 2$, todas uniseriales. Queratodontes diminutos (apenas un poco más grandes que las sierras de los queratostomas). Fil a supraangular más larga que las filas infraangulares.

Coloración en líquido preservativo.- Pardo oscuro dorsal y lateralmente. Ventralmente pardo algoclaro y translúcido. Musculatura caudal pardo rojizopróximamente, pardo uniforme distalmente. Aletas caudales pardo claro uniforme ( $\sin$ manchas), translúcidas.

Comentarios.- Los renacuajos del grupo de Hyla labialis se encuadran dentro del gremio ecomorfológico de "Carnívoro, tipo 1" (Sección II: sistema deaguas no corrientes, Altig \& Johnston 989). La morfología externa (principalmente la parte oral) es ampliamente coincidente con la descripción dada por Altigs \& Johnston (1989) parael gremioecomorfológicoseñaladoy también debido a que este renacuajo es casi idéntico al de Hylameridensis lo que sugiere hábitos igualmente similares a los registrados por Mijares-Urrutia (1990a).

Los caracteres larvarios descritos son ampliamente coincidentes con los conocidos para las larvas de los otros dos miembros del grupo de Hyla labialis y meridensis (Duellman 1989). Las tres especies de Hyla actualmente referidas al grupo de Hyla labialis comparten un número de caracteres que, dentro de Hylidae, la mayoría de ellos son casi ciertamente derivados: fórmula de filasdequeratodontes 1/2ó 2/2, papilas marginales del disco oral en filas múltiples, diastema rostral presente, tubo cloacal diestroseparadode la aleta caudal ventral (abdominal), musculatura caudal alcanza extremo distal de la cola, aletas caudales más altas que la musculaturacaudal,margende la abertura narinal liso con reborde carnoso no protuberante. A pesar de que el autor no examinó directamente renacuajos de $H$. labialis y que la reseñas publicadas del renacuajo de la especie son muy incompletas (Duellman \& Trueb 1982, Duellman 1989, Ladino \& Colmenares 1987), se sabe que al menos si cumple con tres (fórmula de queratodontes en $2 / 2$ filas, diastema rostral presente, aletas caudales más altas que la 
musculatura caudal a la mitad de su longitud) de los siete caracteres larvarios de la lista anterior; los restantes cuatro probablemente también estén presentes en el renacuajo de $H$. labialis, como lo sugiere la estabilidad de los caracteres, enumerados previamente(alolargod elos estadios observados por el autor en las dos especies examinadas), a lo distintivo de los mismos y que a nivel de ejemplares ya adultos el grupo también es muy homogéneo y distintivo en los caracteres que lo definen (Duellman 1989). El estudiode las variaciones de estos caracteres probablemente ayuden a definir las relaciones de las especies incluidas dentro del grupo.

\section{DISCUSIÓN}

Actualmente, todas las publicaciones disponibles sobre listas y características morfológicas larvarias se encuentran en idioma inglés (Altig 1970, Altig \& Johnston 1986, Hero 1990, Johston \& Altig 1986, Lavilla 1988, Van Dijk 1966) no existiendo en idioma castellano versiones traducidas que permitan el empleo de un mismo sistema de términos y definiciones morfoanatómicas único y homogéneo, ni se conoce (al menos por el autor) ninguna proposición independiente de terminología en castellano en este sentido. También cabe agregar que el estudio de la morfología externa larvaria de los anfibios anuros se ve limitada al número de especies estudiadas y las peculiaridades y características propias de las larvas de éstas especies que, como se desprende de la diversidad descrita e ilustrada por Duellman \& Trueb (1986) y por Altig \& Johnston (1986, 1989), la variabilidad es sumamente elevada y existe el convencimiento de que aun faltan por descubrir nuevas combinaciones morfológicas. En consecuencia, la terminología y características morfológicas larvarias descritas de una localidad determinada o de un grupo taxonómico particular empleadas en trabajos previos, no son necesariamente aplicables a todos los subsiguientes estudios en la especialidad.
En este trabajo se propone una terminología en idioma español de la morfología externa como resultado de la observación de numerosos lotes de renacuajos de anuros de los Andes de Venezuela y que pretende revisar, resumir y modificar (en los casos en que se consideró necesario), selectivamente, principalmente a partir de los aportes hechos por Altig (1970), Lavilla (1988) y Van Dijk (1966). Además, una consecuencia adicional resulta de la revisión y traducción de la terminología misma, que es la creacción denuevos términos como, es el caso de el Título V, Numeral 17 , donde se procuran definir ciertos rasgos de la cloaca asociados a las aletas caudales ventrales, $\mathrm{o}$ del Título X, donde se propone precisamente la sustitución del término "Rostrodontes" por "Queratostomas".

Respecto a la trascendencia del estudio de la morfología larvaria de los anfibios anuros se puede visualizar mejor con algunos ejemplos seleccionados. La propuesta de relaciones entre los grupos de especies de Hyla con una carga cromosómica $2 \mathrm{n}=30$ cromosomas, hecha por Duellman \& Trueb (1982), se fundamentó en un análisis en características principalmente larvarias (5 de 8 caracteres analizados). Donnelly et al. (1990), efectuó un análisis cladístico de los géneros incluidos en la subfamilia Microhylinae neotropicales a partir de la comparación de 6 caracteres larvarios. Inger(1992) definióel género Ansonia (un bufónido asiático) a partir de caracteres apomórficos larvarios e intentó un análisis cladístico de las relaciones intragenéricas del género empleando íntegramente caracteres larvarios. La especie Otophrynus robusta cuyo renacuajo axhibe una cantidad y magnitud de modificaciones de caracteres apomórficos no compartidos con ninguna otra larva conocida dentro de la familia Microhylidae, que impulsó a Wassersug \& Pyburn (1987) a proponer la creación de la subfamilia Otophryninae. Finalmente, un ejemplo más completoy detallado de la extensión alcanzada por la radiación adaptativa de los renacuajos la presentan Altig \& Johnston (1989), donde describen en detalle los caracteres morfológicos larvarios asociados a un determinado o a varios ecomorfotipos, dejando 
abierta la posibilidad de que las categorias por ellos se incrementen en la medida que se profundice en el estudio de la morfología y ecología de los renacuajo de grupos poco conocidos (como los de las especies del grupo de Scinax rostrata, los géneros Scarthyla, Hyperolius, Centrolene, entre otros). El estudio de la variabilidad intraespecífica e intragenérica de los distintos ecomorfotipos podría responder muchas incognitas sobre la filogénia, la evolución y la ecología de las especies de anuros.

Debido a que la descripción de especies nuevas de ranas aun continúa (La Marca 1994, "1991" [1994]) y que aun faltan por describirse las larvas de una gran cantidad de especies de anuros de los altos Andes venezolanos, la posibiladad deque se descubran nuevos rasgos morfológicos larvarios o modificaciones novedosas de los ya conocidos, o combinaciones de caracteres no incluidos en esta lista, al menos se aspira a que el presente trabajo sirva como punto de partida para aquellos investigadores de habla hispana interesados en el estudiode las larvas de anfibios anuros andinos y de otras regiones.

\section{AGRADECIMIENTOS}

Expreso mi profunda gratitud a las siguiente personas que estimularon mi interés por la herpetofauna venezolana, que han sidoy continúan siendofuente de aprendizaje, y permanentemente apoyaron mi trabajo, entre otros aspectos, en grata compañía en viajes de recolecta, permitiéndome ocupar espacio en los laboratorios, el acceso a equipos, u of reciendo críticas constructivas, comentarios acertados y consejos oportunos: Alexis Arends, Jaime Aranguren, FranciscoBisbal, Daniel Cabello, Draghitza Díaz, Juan Elías García, Pedro Jiménez, Enrique La Marca, Juan Lorenzo, Angela Martino, Maritza Matta, María N.Ponce, María J. Pradeiro, Ramón Rivero, Osmán Rossell, Samuel Segnini, J.Celsa Señaris, Maricela Sosa (q.e.p.d.),Pascual Soriano, A. Valderrama. También agradezco a F. Bisbal
(Estación Biológica de Rancho Grande, PROFAUNA-MARNR, Maracay), Amelia Díaz y Jaime Péfaur (Colección de Vertebrados, Universidad de Los Andes, Mérida), E. La Marca (Universidad de Los Andes, Colección Herpetológica del Laboratorio de Biogeografía, Mérida), y a M.J. Pradeiro y J. C. Señaris (Museo de Historia Natural La Salle, Caracas) por el acceso al catálogo y préstamo de material depositado en las respectivas colecciones. J. Ayarzagüena, C. Carmona, E. La Marca y J.C. Señaris revisaron ehicieron valiosas críticas sobre versiones preliminares del manuscrito, sin embargo, la responsabilidad sobre el contenido estotalmentedel autor. Deseoexpresarmiespecial reconocimiento a mis profesores en Mérida, Pascual Soriano y Enrique La Marca, y a mis compañeros de estudio, Maricela Sosa (q.e.p.d.) y Juan E. García Pérez, por compartir generosamente conmigo sus amplios conocimientos sobre biologia, experiencias de campo y of recerme constantemente muestras de aprecio y amistad. A ellos dedico, agradecido, este trabajo.

\section{RESUMEN}

Se hizo una revisión de las referencias pertinentes y se examinaron una serie de lotes de renacua jos representativos (al menos una especie por cada género) de los anuros altoandinos de Venezuela. Se presentan también una lista de 41 caracteres con algunas ilustraciones explicativas, intentando describir en detalle la morfología externa larvaria de esos anuros. Se propone emplear la medición de ángulos respecto al eje longitudinal del cuerpo para establecer la forma de la punta de la cola y la ubicación del aparato oral. Igualmente, se tomaron 19 medidas para determinar las proporciones morfológicas. Se incluye una clave para identificar a partir exclusivamente de caracteres morfológicos externos (presencia/ausencia, tamaño, forma, número, ubicación, etc.), loscuales no semodifican (o apenassemodifican ligeramente) a lo largo de los estadios de desarrollo larvario más jóvenes e intermedios. Esta clave sirve para identificar los renacua jos al nivel de familia y género de las ranas y sapos que habitan en la región antes mencionada. La clave abarca seis familias y 11 géneros. Adicionalmente, y como un modelo de este trabajo, se describe e ilustra por primera vez el renacuajo de Hyla pelidna, coincidiendo bastante bien con las características larvarias del grupo de $\mathrm{H}$. labialis (fórmula de filas de queratodontes $1 / 2 \circ 2 / 2$, papilas marginales en filas múltiples, 
diastema rostral presente, abertura cloacal diestra y abdominal [separado de la aleta ventral], aletas caudales más anchas que la musculatura caudal a la mitad de su longitud, abertura narinal con un anillo carnoso, liso, no protuberante). Este trabajo representa el primer esfuerzo para compilar en idioma español la morfología larvaria de los anfibios anuros.

\section{REFERENCIAS}

Altig, R. 1970. A key to the tadpoles of the continental United States and Canada. Herpetologica 26: 180-207.

Altig, R. \& G.F. Johnston. 1986. Major characteristics of freeliving anuran tadpoles. Smiths. Herp.. Info. Serv. 67: 175 .

Altig, R. \& G.F. Johnston. 1989. Guilds of anuran larvae: relationships among developmental modes, morphologies, and habitats. Herp. Monogr. 3: 81-109.

Channing, A. 1984. Identification of ghost frog tadpoles. Proc. Electron Microscopy Soc. S. Afr. 14: 89-90.

Channing, A., R. Boycott \& H.J. van Hensbergen. 1988. Morphological variation of Heleophryne tadpoles from the Cape Province, South Africa (Anura: Heleophrynidae). J. Zool., Lond. 215: 205-216.

Donoso-Barros, R. \& J. León. 1972. Desarrollo y evolución larval de Hyla crepitans (Amphibia-Salientia). Bol. Soc. Biol. Concepción 44: 117-127.

Donnelly, M.A., R.O.de Sá \& C. Guyer. 1990. Description of the tadpoles of Gastrophryne pictiventris and Nelsonophryne aterrima (Anura: Microhylidae), with a review of morphological variation in free-swimming microhylid larvae. Amer. Mus. Novitates 2976: 1-19.

Dubois, A. 1994. Keratodont formulae in anuran tadpoles: proposals for a standardization. J. Zool. Sys. Evol. Res. 32: $297-318$

Duellman, W.E. 1972. A review of the neotropical frogs of the Hyla bogotensis group. Occas. Pap. Mus. Nat. Hist. Univ. Kansas 11: 1-31.

Duellman, W.E. 1978. The biology of an equatorial herpetof auna in Amazonian Ecuador. Univ. Kansas Mus. Nat. Hist. Misc. Publ. 65: 1-352.

Duellman, W.E. 1979. The herpetof auna of the Andes: patterns of distribution, origin, differentiation, and present communities. Monogr. Mus. Nat. Hist. Univ. Kansas 7: 371-459.
Duellman, W.E. 1989. New species of hylid frogs from the Andes of Columbia [sic!] and Venezuela. Occas. Pap. Mus. Nat. Hist. Univ. Kansas 131: 1-12.

Duellman, W.E. \& P. Gray. 1983. Developmental biology and systematics of the egg-brooding hylid frogs, genera Flectonotus and Fritziana. Herpetologica 39: 333-359.

Duellman, W.E. \& L. Trueb. 1982. Frogs of the Hyla columbiana group: taxonomy and phylogenetic relationships, p. 33-51 In: A.G.J. Rhodin \& K. Miyata (eds.). Advances in Herpetology andEvolutionary Biology. Harvard Univ., Cambridge.

Duellman, W.E. \& L. Trueb. 1986. Biology of Amphibians. McGraw-Hill, Nueva York. 670 p.

Echeverría, D.D. 1990. La metamorfosis en los anuros, un cambiocon historia. Asoc. Herpetol. Arg.,ser.divulgación 4: $1-30$.

Gosner, K.L. 1960. A simplified Table for staging anuran embryos and larvae with notes on identification. Herpetologica 16: 183-190.

Hero, J.-M. 1990. An illustrated key to tadpoles occurring in the central amazon rainforest, Manaus, Amazonas, Brazil. Amazoniana 11: 201-262.

Hero, J.-M. \& A. Mijares-Urrutia. 1995. The tadpole of Scinax rostrata (Anura: Hylidae). J. Herpetol. 29: $307-$ 311.

Inger, R.F. 1992. Variation of apomorphic characters in stream-dwelling tadpoles of the bufonid genus Ansonia (Amphibia: Anura). Zool. J. Linnean Soc. 105: 225-237.

Johnston, G.F. \& R. Altig. 1986. Identification characteristics of anuran tadpoles. Herp. Rev. 17: 36-37.

Kenny, J.S. 1969. The amphibians of Trinidad. Studies Fauna Curaçao Caribb. Is., 29(108): 1-78.

Ladino, E. \& I. Colmenaes. 1987. Tabla de desarrollo del estado metamorfico en Hyla labialis. Rev. Fac. Cienc. Univ. Javeriana 1: 85-100.

La Marca, E..1984. A taxonomic and systematic revision of the frogs of the Colostethus collaris group (Anura: Leptodactylidae: Dendrobatinae). Tesis de Maestría, University of Nebraska, Lincoln.

La Marca, E. 1985a. A new species of Colostethus (Anura: Dendrobatidae) from the Cordillera de Mérida, northern Andes, South America. Occas. Pap. Mus. Zool, Univ. Michigan 710: 1-10. 
La Marca, E. 1985b. Systematics and ecological observations on the Neotropical frogs Hylajahni and Hylaplatydactyla. J. Herpetol. 19: 227-237.

La Marca, E. 1986. Description of the tadpole of Ceratophrys calcarata. J. Herpetol. 20: 459-461.

La Marca, E. 1989. A new species of collared frog (Anura: Dendrobatidae: Colostethus) from Serranía de Portuguesa, Andes of Estado Lara, Venezuela. Amphibia-Reptilia 10: 175-183.

La Marca, E. "199l” (1994). Descripción de una género nuevo de ranas (Amphibia:Dendrobatidae) de la Cordillera de Mérida, Venezuela. Univ. Los Andes, Mérida, IGCRN, Anuario de Investigación 1991: 39-41.

La Marca, E. 1992. Catálogo taxonómico, biogeográfico y bibliográfico de las ranas de Venezuela. Cuad. Geogr. Univ. Los Andes 9: 1-197.

La Marca, E. 1994. Taxonomy of the frogs of the genus Mannophryne (Amphibia; Anura: Dendrobatidae). Publ. Soc. Amigos Doñana 4: 1-75.

La Marca, E. 1995. Crisis de biodiversidad en anfibios de Venezuela: estudiodecasos, p. 47-70.En:LaBiodiversidad Neotropical y la Amenaza de lasExtinciones. M.E. Alonzo (ed.). Cuadernos de QuímicaEcológica, $\mathrm{N}^{\circ} 4$, Univ. Los Andes, Mérida.

La Marca, E. \& A. Mijares U. 1988. Description of the tadpole of Colostethus mayorgai (Anura: Dendrobatidae) with preliminary data on the reproductive biology of the species. Bull. Maryland Herp. Soc. 24: 47-57.

Lavilla, E.O. 1988. Lower Telmatobiinae (Anura: Leptodactylidae): generic diagnoses based on larval characters. Occas. Pap. Mus. Nat. Hist. Univ. Kansas 124: $1-19$.

Lavilla, E.O. \& G.J. Scrocchi. 1986. Morfometría larval delos géneros de Telmatobiinae (Anura: Leptodactylidae) de Argentina y Chile. Physis (Buenos Aires), Secc. B 44(106): $39-43$.

Lavilla, E.O. \& M. Rouges. 1992. Reproducción y desarrollo de anuros Argentinos. Asoc. Herpetol. Arg., ser. divulgación 5: 1-66.

Lynch, J.D.. 1986. Origins of the high andean herpetological fauna, p. 478-499. In: High altitude tropical biogeography. F. Vuilleumier \& M. Monasterio (eds.). Oxford University, Oxford.

McDiarmid, R.W. \& R. Altig. "1989-1990" (1990) Description of a bufonid and two hylid tadpoles from western Ecuador. Alytes 8: 51-60.
Mebs, D. 1980. Zur fortpflanzung von Atelopus cruciger (Amphibia: Salientia: Bufonidae). Salamandra 16:65-81.

Mijares-Urrutia, A . 1990a. El renacuajo de Hyla meridensis (Anura: Hylidae) de los Andes de Venezuela. Rev. Biol. Trop. 38: 231-234.

Mijares-Urrutia, A. 1990b. The tadpole of Centrolenella andina (Anura: Centrolenidae). J. Herpetol. 24: 410-412.

Mijares-Urrutia, A. 1991. Descripción del renacuajo de Colostethus leopardalis Rivero con algunos comentarios sobre su historia natural. Amphibia-Reptilia 12: 145-152.

Mijares-Urrutia, A. 1992. El renacuajo de Hyla lascinia con aportes al conocimiento de los renacua jos de H. jahnie $H$. platydactyla (Hylidae) de los Andes Venezolanos. Alytes 10: 91-98.

Mijares-Urrutia, A. \& E. La Marca. 1997. The tadpoles of the genus Nephelobates (Amphibia Anura Dendrobatidae), from Venezuela. Trop. Zool. 10(1): 133-142.

Myers, C.W., A. Paolillo \& J.W. Daly. 1991..Discovery of a defensively malodorous and nocturnal frog in the family Dendrobatidae: Phylogenetic significance of a new genus and species from the Venezuelan Andes. Amer. Mus. 3002: 1-33.

Péfaur, J. \& A. de Pascual. 1982. Aspectos biogeográficos de las comunidades de anfibios y saurios de los Andes Venezolanos, p. 229-261. En:Zoología Neotropical, Actas VIII Congreso Latinoamericano de Zoología. P.J. Salinas (de.). Univ. de Los Andes, Mérida.

Rada, D. 1981. Renacua jos de algunos anfibios de Clarines (Edo. Anzoáteguí, Venezuela). Mem. Soc. Cienc. Nat. La Salle 41(115): 57-76.

Sokol, O.M. 1975. The phylogeny of the anuran larvae: A new look. Copeia 1975(1): 1-23.

Starrett, P. 1960. Descriptions of tadpoles of Middle American frogs. Misc. Publ. Mus. Zool. Univ. Michigan 1 10: 1-37.

Van Dijk, D.E. 1966 Systematic and field keys tothe families, genera, and described species of Southern Africantadpoles. Ann. Natal Mus. 18: 231-286.

Wassersug, R.J. 1973. Aspects of social behavior in anuran larvae, p. 273-297. In: Evolutionary biology of the anuransContemporary research on major problems. J.L. Vial (ed.). Univ. Missouri, Columbia.

Wassersug, R.J. \& W.F. Pyburn. 1987. The biology of the Peret' toad, Otophryne robusta (Microhylidae), with special consideration of its fossorial larva and systematic relationships. Zool. J. Linnean Soc. 91: 137-169. 